Carla Patricia Pico-Cherres; Marcelo Javier Mancheno-Saá; Sonia Fabiola Chaluisa-Chaluisa

http://dx.doi.org/10.35381/r.k.v5i9.667

\title{
Pedidos y transporte: Una unidad estratégica de estudio en indicadores logísticos de la nueva era
}

\section{Orders and transportation: A strategic unit of study in logistic indicators of the new era}

\author{
Carla Patricia Pico-Cherres \\ cpico3047@uta.edu.ec \\ Universidad Técnica de Ambato, Ambato \\ Ecuador \\ https://orcid.org/0000-0003-2143-5444 \\ Marcelo Javier Mancheno-Saá \\ mj.mancheno@uta.edu.ec \\ Universidad Técnica de Ambato, Ambato \\ Ecuador \\ https://orcid.org/0000-0002-8381-0791 \\ Sonia Fabiola Chaluisa-Chaluisa, \\ sf.chaluisa@uta.edu.ec \\ Universidad Técnica de Ambato, Ambato \\ Ecuador \\ https://orcid.org/0000-0002-4816-9986
}

Recibido: 01 de diciembre de 2019

Aprobado: 10 de enero de 2020

\section{RESUMEN}

La presente investigación tiene como objetivo analizar las tendencias principales que comprenden los pedidos y el transporte. Para ello se ha considerado como referencia tres fuentes de análisis: 1) Investigación de Campo, en el sector comercial de la zona 3 del Ecuador. 2) Análisis gráfico descriptivo del mercado 3) Análisis estadístico inferencial mediante la prueba de Friedman. La metodología se enmarcó en un análisis descriptivo explicativo y el instrumento de investigación fue una encuesta aplicado a una muestra conformada de 373 empresas comerciales. Los resultados obtenidos reflejaron un nivel de significancia asintótica de 0.092 mediante la prueba de Friedman para las variables consideradas en comparación con el nivel crítico de 0.05 , lo cual indica que no hay diferencias estadísticas significativas entre las empresas en relación a las dimensiones pedidos y transporte en las 4 provincias seleccionadas. 
Revista Arbitrada Interdisciplinaria KOINONIA

Año V. Vol V. N9. Enero - Junio 2020

Hecho el depósito de Ley: FA2016000010

ISSN: 2542-3088

FUNDACIÓN KOINONIA (F.K). Santa Ana de Coro. Venezuela.

Carla Patricia Pico-Cherres; Marcelo Javier Mancheno-Saá; Sonia Fabiola Chaluisa-Chaluisa

Descriptores: Planificación del transporte; transporte por carretera; comercio interno; planificación estratégica.

\begin{abstract}
The purpose of this research is to analyze the main trends that comprise orders and transportation. For this, three sources of analysis have been considered as reference: 1) Field Research, in the commercial sector of zone 3 of Ecuador. 2) Graphic descriptive analysis of the market 3) Statistical inferential analysis using the Friedman test. The methodology was framed in an explanatory descriptive analysis and the research instrument was a survey applied to a sample made up of 373 commercial companies. The results obtained reflected an asymptotic significance level of 0.092 using the Friedman test for the variables considered compared to the critical level of 0.05 , which indicates that there are no significant statistical differences between the companies in relation to the dimensions of orders and transportation in the 4 selected provinces.
\end{abstract}

Descriptors: Transport planning; road transport; domestic trade; strategic planning.

\title{
INTRODUCCIÓN
}

En la actualidad las empresas se desenvuelven en mercados altamente competitivos debido a que las exigencias tienen estándares más altos en relación al tiempo de entrega, calidad, precio y atención(Costa \& Castaño, 2015).En este contexto se desenvuelven todas las industrias, que han visto en la evolución de las operaciones de pedidos y transporte, actividades esenciales alcanzar estándares de productividad y eficiencia dentro de la logística de cualquier organización.(Galo, Ribeiro, Priscilla, Mergulhaño, \& Vidal, 2018).Cronológicamente desde los años 80 la logística tiene un enfoque cambiante, con una ideología de comunicación y modernización, en 1985 la cadena de valor es introducida y relacionado con la expresión logística, en 1986 se enfoca a los procesos de controlar, planificar el flujo de materia prima, productos terminados, inventario, servicios que abarca la logística del origen al consumo(Rutner, Aviles, \& Cox, 2012).

Así mismo (Narvaez, 2018), destaca que para finales de los noventa, la logística se enfoca en satisfacer los requerimientos de los clientes, la gestión de la cadena de 
Carla Patricia Pico-Cherres; Marcelo Javier Mancheno-Saá; Sonia Fabiola Chaluisa-Chaluisa suministros, implementa, controla y planifica el almacenamiento y flujo de servicio, haciendo referencia a la eficiencia, en el año 2000la logística siente un cambio sustancial para lo cual trata los requerimientos del cliente desde el aprovisionamiento hasta el punto de venta de los productos, donde los materiales son controlados en la mayoría de procesos.(Penabad, Iznaga, \& Rodriguez, 2016).La administración de procesos estratégicos se gestiona en la primera parte de la década, sin embargo, en el año 2004se trata sobre el traslado adecuado de los productos en la cantidad correcta, en el lugar establecido y en el tiempo oportuno.(Encinoso, 2011).La tecnología ha influenciado de manera positiva en el desarrollo eficiente de los procesos logísticos optimizando recursos, no solo este sector ha sido beneficiado, hasta sectores financieros como la banca han empezado su virtualización (Llamuca Pérez, Mancheno Saá, \& Chaluisa Chaluisa, 2019), implantando sistemas informatizados de control en las operaciones, inventarios, identificación codificada de las mercaderías y otros, accediendo con rapidez a la información necesaria (Picón Vizhñay, Erazo Álvarez, \& Narváez Zurita, 2019).

Otro factor importante es la utilización de equipos, transporte y herramientas de gran tecnología asegurando que los productos lleguen a su destino a tiempo y en excelente estado (Mancheno, Villalba, Gamboa, \& Mancheno, 2018). Por otra parte, el uso de indicadores ha jugado un papel importancia en la gestión logística, para determinar las métricas de productividad y eficiencia de las actividades que forman parte del proceso logístico.(Diaz, 2012).

Considerando el Índice de Desempeño Logístico realizado por el Banco Mundial el cual es un análisis donde se mide el rendimiento a lo largo de la cadena logística de suministro dentro de un país, en América Latina en donde el promedio del índice de desempeño logístico elaborado por el Banco Mundial, de los 23 países evaluados en la región tuvo un promedio de 2.66 sobre 5 en el 2018, esto se refleja en la dificultad de tienen los países de la región en relación a la eficiencia, aumento del tiempo y de los costos de intercambio(Banco Mundial, 2018). 
Carla Patricia Pico-Cherres; Marcelo Javier Mancheno-Saá; Sonia Fabiola Chaluisa-Chaluisa

Se según el Ministerio de Transporte (2015), el Ecuador posee un índice de desempeño logístico de 2.78 ubicándose en la posición 62 a nivel mundial, un índice bajo por cuanto la logística en el Ecuador atraviesa una serie de dificultades debidas a los problemas estructurales, posición vehicular, normativas de transporte nacional, esto hace que disminuya la calidad de los servicios prestados, para obtener eficacia en este ámbito se debería tomar en cuenta los medios necesarios como aspectos logísticos para que la transportación sea apta(Manzano Gutierrez, 2019). Al emplear la innovación en las rutas de transporte se quiere tener como resultado la minimización de costos y alcanzar ventajas estratégicas que permiten competir en un mercado globalizado, mediante la aplicación de procesos eficientes, optimizando las rutas de transporte. (Arango, Adarme, \& Zapata, 2010).

La presente investigación pretende dar lineamientos y diagnosticar el estado actual del área de pedidos y transporte en la zona 3 del Ecuador, considerando que la mencionada zona es el centro comercial del país, el diagnostico se realizará a manera de caracterización descriptiva con varios factores, con el fin de conocer de manera holística el término a estudiar.

La Zona 3 del Ecuador incluye las provincias de Chimborazo, Cotopaxi, Pastaza y Tungurahua, zona ubicada en la parte central del país y se ha convertido en una zona estratégica que conecta las regiones de la Costa, Sierra y Amazonia ecuatoriana. (Bravo, Orejuela, \& Osorio, 2007). Para el desarrollo de la investigación se hace referencia a los distintos procedimientos, técnicas,métodos, para tratar de resolver los problemas concernientes a inputs, insumos, materias primas, indicando que la zona es un sector comercial, mayormente referente a comercio de calzado (Mancheno \& Miranda, 2017), donde los pedidos o transporte son imprescindibles y se define actividades de apoyo y actividades principales, enmarcando un mecanismo de desarrollo y defensa empresarial.(Adarme, Fontanilla, \& Arango, 2011) 
Carla Patricia Pico-Cherres; Marcelo Javier Mancheno-Saá; Sonia Fabiola Chaluisa-Chaluisa

\section{METODOLOGÍA}

La investigación es de tipo descriptiva, por cuanto se procuró establecer las características y propiedades del área de pedidos y transporte en la zona 3 del país, siendo importante debido que contribuye en el área comercial y de competitividad(Mancheno Saá \& Albán Bautista, 2019), se convierte en un fenómeno económico que marca el ritmo para el resto del país, en el mencionado análisis, se utilizó variables en base a las dimensiones y variables de pedidos y transporte en una caracterización que estudia la funcionalidad, las características propias de cada variable. Para la determinación de las dimensiones y variables fundamentales se realizó una investigación bibliográfica en revistas de acceso abierto en catálogos Scielo, Redalyc y Google Scholar.

La población está compuesta por 12.584 empresas que se encuentran en la zona 3 del Ecuador, obtenidos de la base de datos del Censo Económico, el diseño del muestreo fue transversal, tomando 4 muestras en las principales ciudades de cada provincia de la zona 3: Ambato, Riobamba, Latacunga y Cotopaxi se consideró una muestra de 373 empresas, el instrumento de investigación fue una encuesta con escala de respuesta a través de escala de Likert, para validar la investigaciónse realizó mediante la aplicación una prueba piloto y método Delphi a través de criterios de expertos en logística y transporte, la confiabilidad mediante Alfa de Cronbachreflejó el 0.87 considerado aceptable según (Campo \& Oviedo, 2008)por cuanto la consistencia interna de una escala se considera aceptable cuando se encuentra entre 0,70 y 0,90.

La recolección de datos se realizó con equipos de encuestadores los cuales procedieron a moverse en campo, dos personas por cada 10 empresas, tomando el muestreo un plazo de 18 meses en el cual se procede a reportar los resultados. El análisis y procesamiento estadístico se realizómediante el software estadístico IBM SPSS versión 21 considerando que los datos provienen de variables ordinales por cuanto se empleó escala de Likert y las muestras provienen de 4 muestras relacionas, 
Carla Patricia Pico-Cherres; Marcelo Javier Mancheno-Saá; Sonia Fabiola Chaluisa-Chaluisa se seleccionó la prueba estadística no paramétrica de Friedmanpara examinar si las variables comparten la misma distribución de su origen considerando significación estadística para $p \leq 0,05$.(Berlanga \& Rubio, 2012).

\section{RESULTADOS}

\section{Revisión literaria}

\section{Tabla 1}

Revisión literaria

Variables

$\begin{array}{lrll}\text { Políticas } & \text { y } & \text { La preparación de pedidos es un aspecto importante } \\ \text { procedimientos de } & \text { dentro de cualquier almacén, en comparación con las } \\ \text { preparación } & \text { de } & \text { etapas de envío, almacenamiento y recepción, tiene un } \\ \text { pedidos } & \text { y } & \text { impacto directo en los niveles de satisfacción del cliente. }\end{array}$

Identificación de Proceso en el cual se clasifica y categoriza (Sanjay \& Ravi, mercaderías lasmercaderías, se recomienda evaluar las 2007) características de los productos, la unidad de almacenamiento, los elementos 0 equipos de manipulación, los costos de operación y las TIC disponibles para la identificación y ubicación.

Lista de pedidos La lista de pedidos es un documento que incluye (Baldi, 2019) detalles sobre el contenido específico del tipo y cantidad de cada mercadería solicitada.

Sistema de Un sistema de gestión de inventario es la combinación (Astanti, 2018) inventario de tecnología hardware y software, procesos y (Mancheno, procedimientos que supervisan el monitoreo y Villalba, mantenimiento de los productos almacenados, ya sean Gamboa, \& productos de la compañía, materias primas y Mancheno, suministros, o productos terminados listos para ser 2018) enviados a proveedores o consumidores finales. 
Carla Patricia Pico-Cherres; Marcelo Javier Mancheno-Saá; Sonia Fabiola Chaluisa-Chaluisa

Operaciones

Transporte

mercadería
Se llevan a cabo para administrar las existencias de

(Castillo, 2017) inventario en relación a pedidos múltiples y de reabastecimiento de inventario continuo, que tienen formas distintas de activar órdenes de suministro y equilibrar la demanda con las órdenes de producción y compra y existencias de inventario óptimas para evitar quedarse sin existencias.

de El envío de carga es clave para llevar las mercancías a un destino a tiempo, de manera segura y rentable.
(Manotas

$\&$

Vizcaíno, 2010)

Fuente: Investigadores a partir de autores citados en tabla 1.

\section{Investigación de Campo}

\section{Grafico 1}

Preparación de pedidos

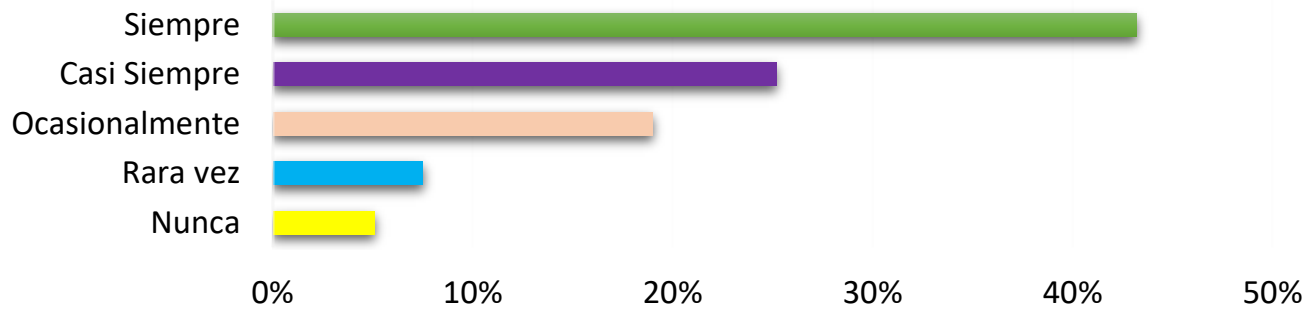

Fuente: Autores, 2019

El $5 \%$ afirman que nunca establecen políticas o procedimientos de preparación de pedidos, seguido del $8 \%$ que corresponde a rara vez, ocasionalmente el 19\%, casi siempre el $25 \%$, y finalmente el $43 \%$ correspondiente a siempre, dando como perspectiva que en la zona tres del Ecuador que la mayor parte sigue política de preparación de pedidos. 


\section{Grafico 2}

Identificación de mercaderías

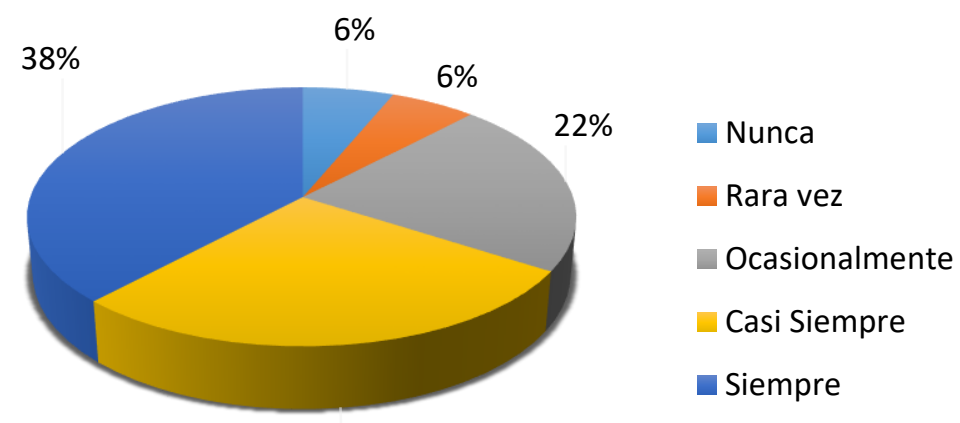

$28 \%$

Fuente: Autores, 2019

El $6 \%$ afirman que nunca se identifican las mercaderías con códigos de barra, cantidades, lote, nombre, fecha de caducidad y características, el 6\% corresponde a rara vez, ocasionalmente el $22 \%$, casi siempre el $28 \%$ y finalmente el $38 \%$ correspondiente a siempre, dando como resultado que en la zona tres del Ecuador la mayor parte de mercaderías son identificadas mediante los métodos anteriormente mencionados. 


\section{Gráfico 3}

Listado de pedidos

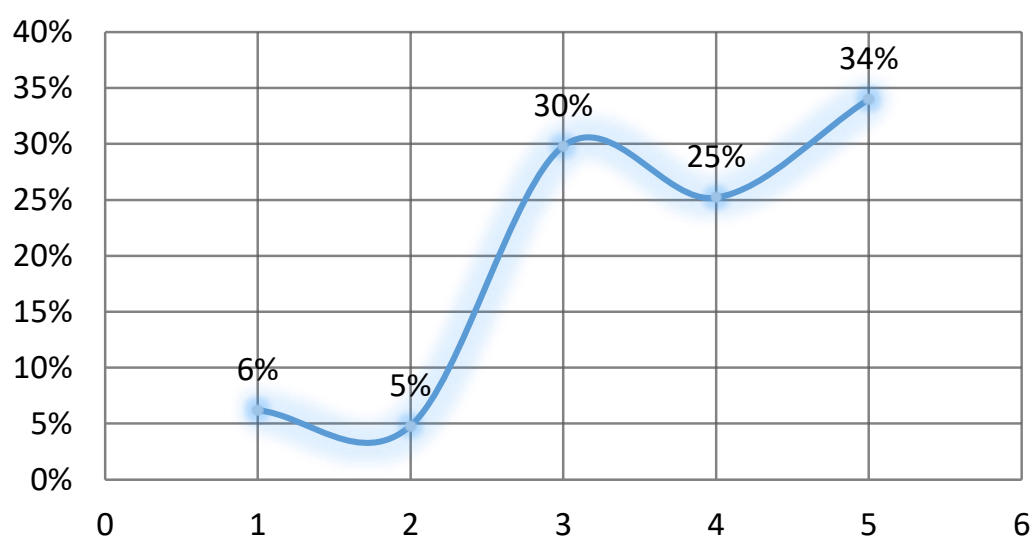

Fuente: Autores, 2019

El 6\% afirman que nunca poseen el listado de pedidos de clientes de forma manual o informatizada, seguido del $5 \%$ que corresponde a rara vez, ocasionalmente al $30 \%$, casi siempre el $25 \%$ y finalmente el $34 \%$ correspondiente a siempre, dando como perspectiva que en la zona tres del Ecuador la mayor parte de encuestados posee lo anteriormente mencionado. 


\section{Grafico 4}

Sistema de inventarios

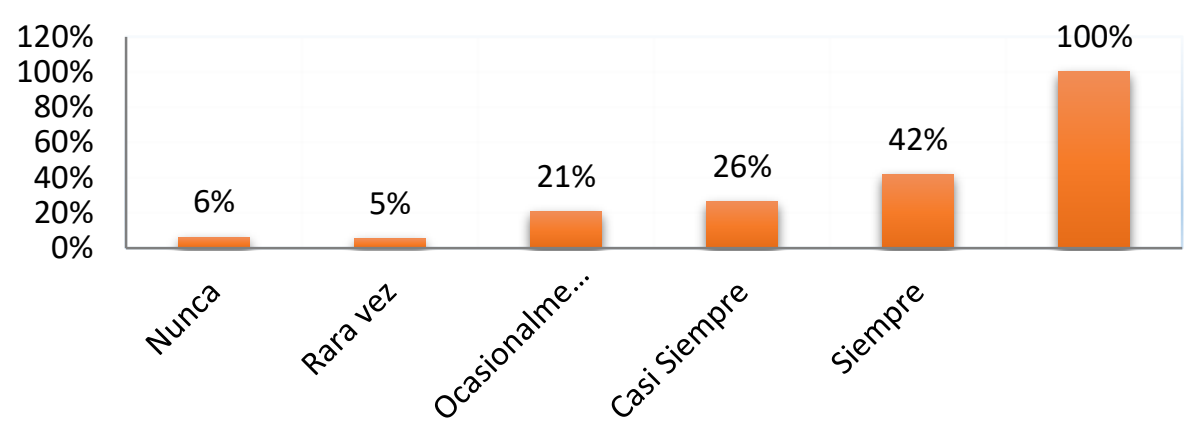

Fuente: Autores, 2019

El 6\% afirman que nunca se mantiene un sistema de inventario manual o informatizado, seguido del $5 \%$ que corresponde a rara vez, ocasionalmente el $21 \%$, casi siempre el $26 \%$ yfinalmente el $42 \%$ correspondiente a siempre deduciendo que en la zona tres del Ecuador la mayor parte de encuestados sigue manteniendo un sistema anteriormente mencionado. 


\section{Grafico 5}

Operaciones

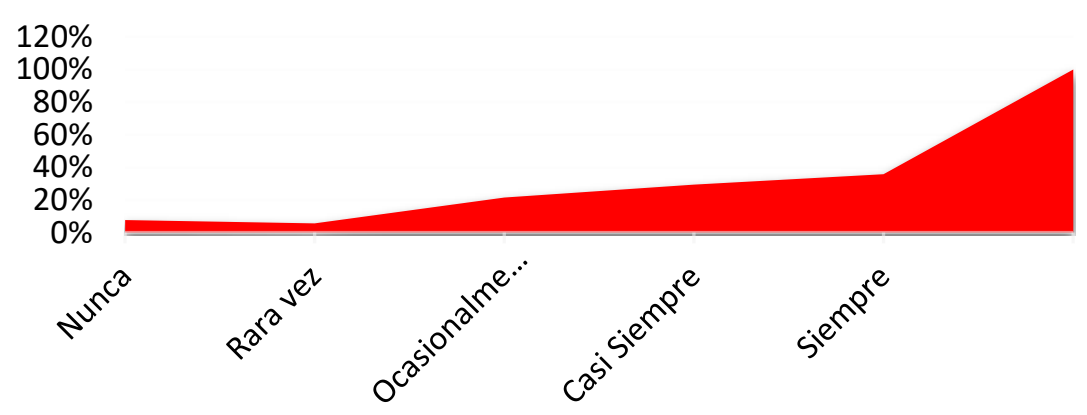

Fuente: Autores, 2019

El 8\% afirman que las operaciones de manipulación no provocan interrupciones en la preparación de pedidos, seguido del $6 \%$ que corresponde a rara vez, ocasionalmente el $21 \%$, casi siempre el $30 \%$ y finalmente el $36 \%$ correspondiente a siempre, dando como perspectiva que en la zona tres del Ecuador la mayor parte de las operaciones de manipulación no provocan interrupciones en la preparación de pedidos. 


\title{
Grafico 5
}

Acceso rápido a mercaderías

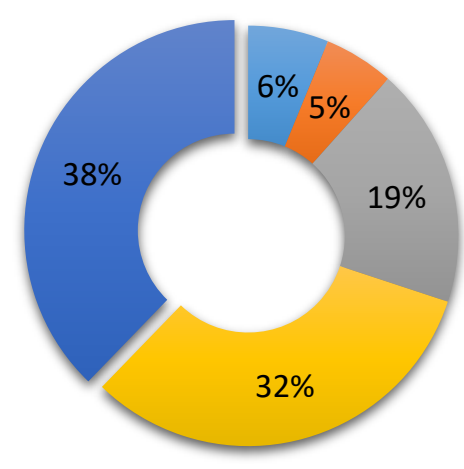

\author{
Nunca \\ - Rara vez \\ Ocasionalmente \\ Casi Siempre \\ - Siempre
}

Fuente: Autores, 2019

El $6 \%$ afirma que se accede rápidamente a la mercadería solicitada, seguido del $5 \%$ que corresponde a rara vez, ocasionalmente el $19 \%$, casi siempre el $32 \%$ y finalmente el $38 \%$ correspondiente a siempre, arrojando los siguientes resultados donde la mayoría accede rápidamente a la mercadería solicitada en la zona tres del Ecuador 


\section{Grafico 6}

Área de despacho y transporte

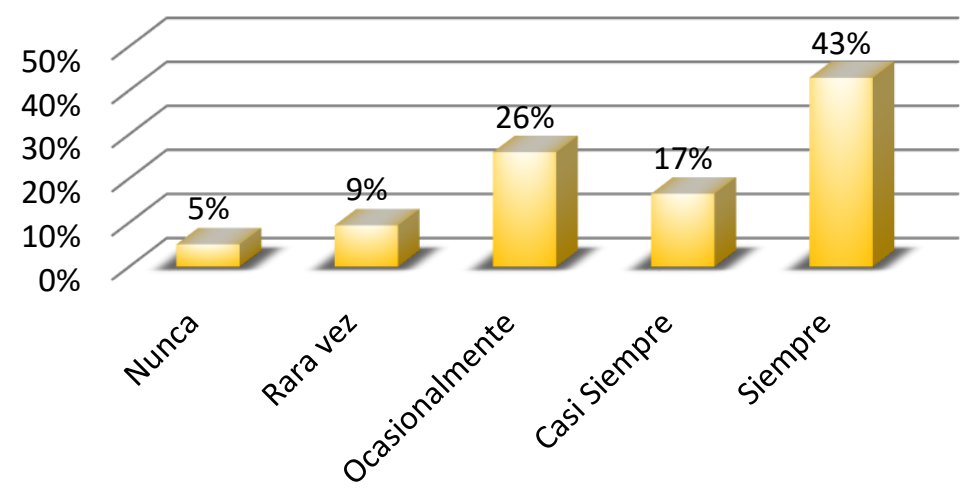

Fuente: Autores, 2019

El 5\% afirman que nunca han contado con un área de despacho y transporte., seguido del $9 \%$ que corresponde a rara vez, ocasionalmente el $26 \%$, casi siempre el $17 \%$ y finalmente el $43 \%$ correspondiente a siempre, dando como perspectiva que la mayor parte cuenta con un área anteriormente mencionada en la zona tres del Ecuador. 


\section{Grafico 7}

Embarque y transporte

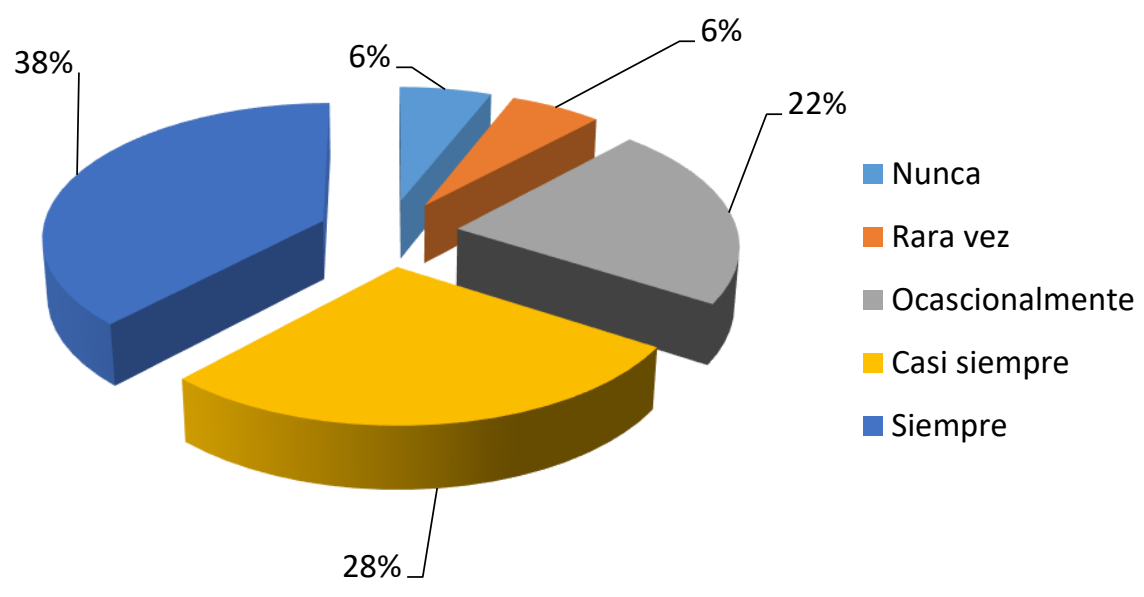

Fuente: Autores, 2019

El $5 \%$ afirman que nunca el embarque y transporte protege, garantiza, la integridad física de la mercadería, seguido del $6 \%$ que corresponde a rara vez, ocasionalmente el $22 \%$, casi siempre el $28 \%$ y finalmente el $38 \%$ correspondiente a siempre, dando como perspectiva que en la zona tres del Ecuador la mayor parte de encuestados observan que la mercadería está protegida y garantizada en el embarque y transporte. 


\section{Prueba de Friedman}

\section{Tabla 2}

Rangos

Variables

-Políticas o procedimientos de preparación de pedidos. -Las mercaderías se encuentran identificadas con códigos de barra, cantidades, lote, nombre, fecha de caducidad y características -Se tiene el listado de pedidos de clientes de forma manual o informatizada

-Se mantiene un sistema de inventario manual o informatizado. -Las operaciones de manipulación no provocan interrupciones en la preparación de pedidos.

-Se cuenta con un área de despacho y transporte.

-El embarque y transporte protege y garantiza la integridad física de la mercadería.

Fuente: Autores, 2019

\section{Tabla 3}

Prueba de Friedman
Rangos

5,18

4,97

4,71

5,07

4,80

4,82

5,00

\section{Test Statistics ${ }^{a}$}

\begin{tabular}{lr}
\hline $\mathrm{N}$ & 373 \\
Chi-Square & 10,888 \\
$\mathrm{df}$ & 6 \\
Asymp. Sig. &, 092 \\
a. Friedman Test & \\
\hline
\end{tabular}


Carla Patricia Pico-Cherres; Marcelo Javier Mancheno-Saá; Sonia Fabiola Chaluisa-Chaluisa

\section{Análisis}

El estadístico de Friedman se distribuye según el modelo de probabilidad Chi-cuadrado con $\mathrm{n}$ grados de libertad y se utiliza para comparar promedios poblacionales cuando se trabaja con muestras relacionadas (Ramírez, 2014) .

El valor p o también conocido como significancia demenor o igual $(\leq)$ a 0,05 para rechazar la hipótesis nula, se debe indicar que el nivel de significancia que se establece en ciencias sociales y en psicología normalmente es 0.05 .

Con un nivel de significancia asintótica de 0.092 mediante la prueba de Friedman para las variables consideradas en comparación con el nivel crítico de 0.05 es mayor por lo tanto se mantiene la hipótesis nula, en el cual se indica que no hay diferencias estadísticas significativas entre las empresas en relación a las dimensiones pedidos y transporte en las 4 provincias seleccionadas, por lo cual las dimensiones y sus variables son similares y los indicadores que se presentan a continuación pueden ser útiles en cualquier empresa de las provincias de la zona 3 del Ecuador.

\section{Tabla 4}

Indicadores

Indicadores

Políticas y

procedimientos de

preparación de

pedidos y

transporte

Identificación de

mercaderías

Lista de pedidos

Sistema de

inventario

Operaciones

\section{Calculo}

$\frac{\text { Politicas relacionas a preparacion de pedidos y transporte }}{\text { Politicas establecidas }} \times 100$

$\frac{\text { Mercaderías identificadas con códigos }}{\text { Total de mercaderías }} \times 100$

$\frac{\text { Pedidos entregados con éxito }}{\text { Pedidos totales }} \times 100$

Mercaderias ingresadas en el sistema de inventarios Toal de mercaderias

$\underline{\text { Operaciones de pedidos y transporte realizados con éxito }} \times 100$ 
Carla Patricia Pico-Cherres; Marcelo Javier Mancheno-Saá; Sonia Fabiola Chaluisa-Chaluisa

Transporte de mercadería $\frac{\text { Pedidos transportados con éxito }}{\text { Total de pedidos transportados }} \times 100$

Fuente: Autores, 2019

\section{CONCLUSIONES}

En la revisión literaria en la tabla 1, se puede evidenciar varios factores referentes a la logística comercial, y específicamente con los pedidos y transporte, así las categorías referidas son: Almacenamiento, recepción, satisfacción del consumidor, TICS para identificación y ubicación, lista de pedidos, sistema de inventario, operaciones y transporte de mercadería. De esta forma quedando definida la lista de categorías de estudio para el área de pedidos y transporte.

En la actualidad se realizan estudios acerca del establecimiento de indicadores para medir la incidencia de los distintos factores que influyen en la eficacia y eficiencia de la cadena comercial de suministro, esta a su vez, engloba los procesos logísticos donde es determinante emplear un sistema apropiado de indicadores para obtener resultados y en base a este, poder medir y diagnosticar la gestión para alcanzar eficiencia a lo largo de toda la cadena de suministros, minimizando costos y acelerando la respuesta al mercado.

El transporte tiene como objetivo cubrir las necesidades de un mercado creciente y altamente demandante, a través de la superación de obstáculos, físicos, de tiempo y espacio que surgen al intercambiar la mercancía y en las relaciones personales. El transporte por carretera tiene mayor confiabilidad con menos pérdidas y daños, siendo para los empresarios una gran ventaja con un valor importante.

La realización de mejoras dentro de los servicios de distribución de los productos, impacta de forma significativa en las características del servicio de transporte, alcanzando los objetivos complementarios mediante un sistema. La optimización de los costes de transporte es un proceso complejo para lo cual es indispensable poseer un apropiado sistema de información, recursos necesarios e infraestructura. 
Carla Patricia Pico-Cherres; Marcelo Javier Mancheno-Saá; Sonia Fabiola Chaluisa-Chaluisa

El transporte en la actualidad sufre una revolución autentica, principalmente causada por la tecnología que son potenciados por las TIC en el sistema de transporte(Mancheno, Salinas, Miranda, \& Yugcha, 2018), que lo hace más integrados, seguros, inteligentes y eficientes. Esta revolución pretende disminuir conflictos relacionados con la saturación de las rutas principales y medios de transporte que operan con sus capacidades al límite, causando retrasos y atascos.

La logística es una expresión tradicional que ha ido evolucionando con el transcurrir del tiempo, y relacionándose con otras ciencias,ampliando su horizonte e intentando implicar la problemática presente en la comercialización que realizan las organizaciones.

La logística presenta una caracterización percepcional, pudiendo cambiar desde tendencias distintas que están relacionadas con la misma, sin embargo, existen fundamentales y distintos pilares que ayudaran para su apoyo y desarrollo, a través del tiempo los conceptos modernistas se van diferenciando con la tendencia del momento.

Se recomienda el uso de los indicadores establecidos en la tabla 4, el cual indica que cada uno de los factores deberán cuantificarse a través de indicadores a manera de ratios para que el área de estudio no quede en la subjetividad, a esto se suma la prueba de Friedman donde se demostró que no existe diferencias significativas las dimensiones: preparación de pedidos y transporte entre las empresas de la zona 3 del Ecuador.

\section{REFERENCIAS CONSULTADAS}

1. Adarme, W., Fontanilla, C., \& Arango, M. (2011). Modelos logísticos para la optimización del transporte de racimos de fruto fresco de palma de aceite en Colombia. [Logistic models for the optimization of the transport of fresh oil palm bunches products in Colombia]. Redalyc, 89-113. Recuperado de https://www.redalyc.org/articulo.oa? $\mathrm{id}=91122651006 \& \mathrm{idp}=1 \& \mathrm{cid}=431413$

2. Arango, M., Adarme, W., \& Zapata, J. (2010). Gestión cadena de abastecimiento - logística con indicadores bajo incertidumbre, caso aplicado sector panificador Palmira. [Supply chain management - logistics with indicators under uncertainty, 
Carla Patricia Pico-Cherres; Marcelo Javier Mancheno-Saá; Sonia Fabiola Chaluisa-Chaluisa

applied. Case applied to Palmira bakery sector]. Redalyc, 97-15. Obtenido de https://www.redalyc.org/articulo.oa?id=91114807007

3. Astanti, R. D. (2018). Two techniques for solving nonlinear decreasing demand inventory system with shortage backorders. [Dos técnicas para resolver el sistema de inventario de demanda decreciente no lineal con almacenamiento de pedidos]. International Journal of Operational Research.https://doi.org/10.1504/IJOR.2018.089129

4. Baldi, Mauro Maria\&Manerba, Daniele\&Perboli, Guido \&Tadei, Roberto, 2019. "A Generalized Bin Packing Problem for parcel delivery in last-mile logistics." ["Un problema generalizado de embalaje de contenedores para la entrega de paquetes en logística de última milla"]. European Journal of Operational Research, Elsevier, vol. 274(3), pages 990-999. DOI: 10.1016/j.ejor.2018.10.056

5. Banco Mundial. (2018). De las partes a los productos: por qué la logística comercial es importante. [From parts to products: why business logistics is important]. Recuperado https://www.bancomundial.org/es/news/feature/2018/07/24/from-parts-toproducts-why-trade-logistics-matter

6. Berlanga-Silvente, V., \& Rubio-Hurtado, M. (2012). Clasificación de pruebas no paramétricas. Cómo aplicarlas en SPSS. [Classification of non-parametric tests. How to apply them in SPSS]. REIRE Revista d'Innovació i Recerca en Educació, 5(2), 101-113. https://doi.org/10.1344/reire2012.5.2528

7. Bravo, J., Orejuela, P., \& Osorio, C. (2007). Administración de recursos de distribución: Indicadores para la priorización en transporte Estudios Gerenciales. [Management of distribution resources: Indicators for prioritization in transportation Management Studies]. Redalyc, 101-118. Obtenido de https://www.redalyc.org/articulo.oa?id=21210204

8. Campo, A., \& Oviedo, H. (2008). Propiedades Psicométricas de una Escala: la Consistencia Interna. [Psychometric Properties of a Scale: Internal Consistency]. Revista de Salud Pública, 831-839. Recuperado de https://www.scielosp.org/article/rsap/2008.v10n5/831-839/\#ModalArticles

9. Carvajal, L., \& García, J. (2014). Preparación de pedidos y venta de productos. [Preparation of orders and sale of products]. Madrid: Editorial EDITEX.

10. Castillo, C. F. (2017). Sistema de administración de operaciones para el sector frutícola del cantón Cevallos provincia de Tungurahua. [Operations management 
Carla Patricia Pico-Cherres; Marcelo Javier Mancheno-Saá; Sonia Fabiola Chaluisa-Chaluisa

system for the fruit sector of the Cevallos canton, Tungurahua province]. Ambato: Universidad Técnica de Ambato, Facultad de Ciencias Administrativas.

11. Costa, Y., \& Castaño, N. (2015). Simulación y optimización para dimensionar la flota de vehículos en operaciones logísticas de abastecimiento- distribución. [Simulation and optimization to size the vehicle fleet in supply-distribution logistics operations]. Scielo, $\quad 3 . \quad$ Obtenido de https://scielo.conicyt.cl/scielo.php?script=sci arttext\&pid=S0718$\underline{33052015000300006 \& l a n g=e s}$

12. Díaz, Y. (2012). "La logística empresarial y la administración de inventario" en Observatorio de la Economía Latinoamericana. 168. ["Business logistics and inventory management" at the Observatory of the Latin American Economy. 168]. Recuperado de http://www.eumed.net/cursecon/ecolat/cu/2012/ydc.html

13. Encinoso, L. (2011). La informática en la dirección de empresas. [Computer science in business management]. Cuba: Féliz Varela.

14. Galo, N., Ribeiro, Priscilla, Mergulhaño, R., \& Vidal, J. (2018). Selección de proveedor de servicios logísticos: Alineación entre criterios e indicadores. [Selection of logistics service provider: Alignment between criteria and indicators]. Scielo, 2. Obtenido de http://www.scielo.org.co/scielo.php?script=sci arttext\&pid=S0121$\underline{50512018000300055 \& \text { lang }=\mathrm{es}}$

15. González, A., \& García, M. (2016). Diseño de un sistema de información de indicadores logísticos. [Design of a logistics indicator information system]. Redalyc, 37-50. Recuperado de https://www.redalyc.org/jatsRepo/3579/357947335003/index.html

16. Llamuca-Pérez, S. L., Mancheno-Saá, M. J., \&Chaulisa-Chaluisa, S. (2019). Ebanking, una necesidad de virtualización en el sector financiero ecuatoriano. [Ebanking, a need for virtualization in the Ecuadorian financial sector]. Revista Científica FIPCAEC (Fomento de la investigación y publicación en Ciencias Administrativas, Económicas y Contables). ISSN: 2588-090X. Polo de Capacitación, Investigación y Publicación (POCAIP), 4(4), 578-594.

17. Mancheno, M. J., \& Miranda, R. F. V. (2017). Habilidades directivas y el desarrollo empresarial en la industria de calzado a través de la modelización. [Management skills and business development in the footwear industry through modeling]. Revista Publicando, 3(9), 620-637. 
Carla Patricia Pico-Cherres; Marcelo Javier Mancheno-Saá; Sonia Fabiola Chaluisa-Chaluisa

18. Mancheno, M. J., Salinas, J. M. G., Miranda, R. F. V., \&Yugcha, J. D. P. H. (2018). Caracterización de la logística comercial y su evolución. [Characterization of commercial logistics and its evolution]. Revista Publicando, 5(15 (2)), 817-833.

19. Mancheno, M. J., Villalba, R., Gamboa, J., \&Mancheno, J. (2018). Logística Comercial. [Commercial logistics]. Revisión Literaria. Polo del Conocimiento, 3(10), 465-483. Obtenido de http://polodelconocimiento.com/ojs/index.php/es/article/view/785/985

20. Mancheno-Saá, M. J., \& Albán-Bautista, M. L. (2019). Competitividad Sistémica Empresarial, un término que define el rendimiento de mercados modernos. [Systemic Business Competitiveness, a term that defines the performance of modern markets]. Revista Científica FIPCAEC (Fomento de la investigación y publicación en Ciencias Administrativas, Económicas y Contables). ISSN: 2588090X. Polo de Capacitación, Investigación y Publicación (POCAIP), 4(4), 559577.

21. Manotas, V., \& Gómez Vizcaíno, L. S. (2010). Stochastic programming models for designing logistic networks. [Modelos de programación estocástica para el diseño de redes logísticas]. Revista PROSPECTIVA, 13-19.

22. Manzano Gutiérrez, M. A. (2019). Logística comercial: un enfoque para la toma de decisiones en las MIPYMES de la Zona 3 del Ecuador. [Commercial logistics: an approach to decision making in MSMEs in Zone 3 of Ecuador].

23. MinisteriodeTransporte. (2015). Caracterización del Transporte en Colombia, Diagnóstico y Proyectos de Transporte e Infraestructura. [Characterization of Transportation in Colombia, Diagnosis and Transportation and Infrastructure Projects]. Bogotá. $\quad$ Recuperado de https://www.mintransporte.gov.co/documentos/15/estadisticas/

24. Narvaez, C. (2018). Importancia de la Administración de Procesos de Negocio para el Área de Transportes de los Operadores Logísticos Industrial. [Importance of Business Process Administration for the Transport Area of Industrial Logistics Operators]. Redalyc, 113-121. Obtenido de https://www.redalyc.org/articulo.oa?id=81658059016

25. Picón Vizhñay, J., Erazo Álvarez, J., \& Narváez Zurita, C. (2019). Plan de expansión empresarial para la Empresa Transnexos del Grupo Industrial Graiman. [Business expansion plan for the Transnexos Company of the Graiman Industrial Group]. Revista Arbitrada Interdisciplinaria Koinonía, 4(1), 274-308. http://dx.doi.org/10.35381/r.k.v4i1.459 
Carla Patricia Pico-Cherres; Marcelo Javier Mancheno-Saá; Sonia Fabiola Chaluisa-Chaluisa

26. Penabad, L., Iznaga, A., \&Rodríguez, P. (2016). Disposición y disponibilidad como indicadores para el transporte. [Disposition and availability as indicators for transport]. Redalyc, 64-73. https://www.redalyc.org/articulo.oa?id=93249315008

27. Ramírez, J. (2014). Análisis de aceptación y preferencia del manjar blanco del valle. [Analysis of acceptance and preference of the white delicacy of the valley]. Biotecnología en el Sector Agropecuario y Agroindustrial, 20-27.

28. Rutner, S., Aviles, M., \& Cox, S. (2012). Logistics evolution: a comparison of military and commercial logistics thought. [Evolución logística: una comparación del pensamiento logístico militar y comercial]. International Journal of Logistics Management, 96-118.

29. Sanjay, J., \& Ravi, S. (2007). Selection of logistics service provider: An analytic network process (ANP) approach. [Selección del proveedor de servicios logísticos: un enfoque de proceso de red analítica (ANP)]. Omega, 274-289. 